\title{
Development of Future EU District Heating and Cooling Network Solutions, Sharing Experiences and Fostering Collaborations ${ }^{\dagger}$
}

\author{
Andrea Costa ${ }^{1}$, Tatiana Loureiro 1,*, Francesco Passerini ${ }^{1}$, Susana Lopez ${ }^{2}$, Dirk Pietrushka ${ }^{3}$, \\ Martin Klepal ${ }^{4}$, George Halikias ${ }^{5}$, Ioannis Meintanis 5, Serafeim Moustakidis ${ }^{5}$, \\ Nikos Karcanias ${ }^{5}$, Martin Bucholz ${ }^{6}$ and Inés Arias ${ }^{7}$ \\ 1 R2M solution srl, Via Fratelli Cuzio, 42, 27100 Pavia PV, Italy; andrea.costa@r2msolution.com (A.C.); \\ francesco.passerini@r2msolution.com (F.P.) \\ 2 IK4-Tekniker, Calle Iñaki Goenaga, 5, 20600 Eibar, Gipuzkoa, Spain; susana.lopez@tekniker.es \\ 3 Enysist GmbH, Robert-Bosch-Str. 8/1, 72124 Pliezhausen, Germany; dirk.pietruschka@enisyst.de \\ 4 Cork Institute of Technology, Rossa Avenue, Bishopstown, T12 P928 Cork, Ireland; martin.klepal@cit.ie \\ 5 City University London, Northampton Square, Clerkenwell, EC1V 0HB London, UK; \\ g.halikias@city.ac.uk (G.H.); ioannis.meintanis.1@city.ac.uk (I.M.); \\ serafeim.moustakidis.1@city.ac.uk (S.M.); n.karcanias@city.ac.uk (N.K.) \\ 6 Watergy GmbH, Oderberger Str. 3, 10435 Berlin, Germany; martin.buchholz@watergy.de \\ 7 Euroheat \& Power, Cours Saint Michel 30a-Box E, 1040 Brussels, Belgium; ia@euroheat.org \\ * Correspondence: tatiana.loureiro@r2msolution.com; Tel.: +34-644-966-949 \\ † Presented at the Sustainable Places 2017 (SP2017) Conference, Middlesbrough, UK, 28-30 June 2017.
}

Published: 13 November 2017

\begin{abstract}
Heating and cooling consume half of the EU's energy and much of it is wasted. The lion's share of heating and cooling is still generated from fossil fuels, mainly natural gas, while only $18 \%$ is generated from renewable energy. In order to fulfil the EU's climate and energy goals, the heating and cooling sector must therefore sharply reduce its energy consumption and cut its use of fossil fuels. To this end the European Commission adopted a heating and cooling strategy in February 2016 as part of the wider Energy Union Package. A number of activities and projects funded by the programmes of European Union are supporting this new EU heating and cooling strategy.
\end{abstract}

Keywords: heating and cooling; district energy; district heating and cooling; INDIGO; FLEXYNETS; E2District; InDeal; H-Disnet; SDHp2m

\section{Introduction}

The European Commission's heating and cooling strategy makes clearly the case that demand reduction and the deployment of renewable energy and other sustainable sources, such as waste heat, have a great potential to reduce fossil fuel import and ensure energy supply security, while ensuring affordable provision of energy for the end consumer [1]. Some of the projects presented at this workshop deal with adaptive control and optimisation methods. The control systems can operate at different levels: production, distribution, storage, HVAC systems, demand side (e.g., demand response systems). The control systems can use the results of mathematical simulations and their goals can be the demand satisfaction, the reduction of the energy demand, the reduction of the costs. Other of the introduced projects deal with low-temperature networks that are used both for heating and for cooling (DHC networks). The connection to those networks may be through reversible electric heat pumps or through absorption/desorption phenomena. Another goal of the projects here described is the dissemination of the know-how relative to the district heating and to the district cooling systems. A method can of design tools that facilitate the designers' work. 


\section{INDIGO}

The main objective of INDIGO project is the development of a more efficient, intelligent, and cheaper generation of DC systems by improving system planning, control, and management. Specific Objective 1: To increase DC systems efficiency, thus contributing to a reduction over $45 \%$ of the primary energy consumption and to a reduction in Europe by 2030 of more than 2.5 million tons of $\mathrm{CO}_{2}$ emissions, for space cooling trough DC systems compared to today's level. Specific Objective 2: To validate in a real District Heating and Cooling (DHC) installation the benefits of INDIGO developments, and to use this case-study as an exploitation demonstrator of INDIGO new generation of DC systems. The INDIGO project involves the development of highly efficient and intelligent DC systems based on: an innovative and optimized DC system Management Strategy; the integration of predictive controllers at component level, some of them including self-learning algorithms for accuracy improvement. The controllers are based on reduced models, which are the reduction of physical models. An open-source library with parametric thermo-fluid dynamic models of District Cooling System components is one of the main results of INDIGO [2]. Besides, open source tools and guidelines will be developed within the project in order to provide more confidence and thus more openness to DC systems development and use [3].

\section{FLEXYNETS}

Classical District Heating and Cooling (DHC) networks distribute energy from a centralized generation plant to a number of remote customers. As such, actual DHC systems suffer from significant heat losses, highly unexplored integration potential of different available energy sources (e.g., Renewables and waste heat) into the network and high installation costs. FLEXYNETS will develop, demonstrate and deploy a new generation of intelligent district heating and cooling networks that reduce energy transportation losses by working at "neutral" temperature levels (10$20^{\circ} \mathrm{C}$ ). Reversible heat pumps and chillers will be used to exchange heat with the DHC network on the demand side. In this way, the same network can provide contemporary heating and cooling. FLEXYNETS solutions will integrate effectively multiple generation sources (including high- and low-temperature solar thermal, biomass, PV, cogeneration and waste heat) where they are available along the DHC network, by managing energy at different temperature levels and assuring optimized exergy exploitation. Together with storages, control strategies that optimize the harvest of renewable energy sources are key from the technical and economic points of view. On the one hand, strategies will be assessed that assure a thermal balance among diffused heat generation, storage and utilization. On the other, policies will be elaborated to decide when energy is to be gathered locally or exchanged (both purchased and sold) with the electricity and gas networks. The optimal management of such new generation networks will lead to a synergic effect on primary energy savings (hence on the reduction of the $\mathrm{CO} 2$ emissions), assuring at the same time investment and operation profitability. As such, FLEXYNETS will contribute to a higher penetration of smart DHC networks on the heating and cooling market, and will contribute to the European recovery plan. To test and analyze developed control strategies and systems an outdoor pilot plant has been built at EURAC in Bolzano as small scale DHC loop including different single devices (ORC unit, concentrating solar collectors, sorption HP, compression HP). Within this loop, a number of distributed prosumers are emulated by two reversible heat pumps. With this laboratory system it will be possible to emulate a set of independent simulated sources, users and prosumers along a network. Simulation studies are used to analyse the implementation of the FLEXYNETS concept in the built environment. Combined energetic and economic analyses on typical case studies will highlight most promising application potentials for a future market roll out of the innovative low exergy DHC network technology [4]. 


\section{E2District}

The focus of E2District is to develop an innovative cloud based software infrastructure and people engagement strategy to manage and support smart energy usage and decision making for DHC systems. E2District's key goals are: (i) Optimising DHC production and distribution asset usage, (ii) Analysing and influencing behavioural demand, (iii) Developing new DHC business models to achieve energy efficient districts, and (iv) Demonstrating and evaluating an innovative E2District Operations system E2District aims to make DHC systems more efficient, intelligent and cheaper. The project is targeting energy cost savings of 30\%, indicating that eventual widespread adoption of similar smart district heating and cooling infrastructures could deliver significant efficiencies and reduced costs over the long-term. To achieve these goals, not only is it necessary that advancements are made on the technical side but, also, end-user behaviour has to be integrated into the whole system. The project is creating a replicable system that can be adapted for other similarly managed industrial and domestic sites - whether local, national or international. According to Heat Roadmap Europe, DH and CHP will fulfil 30\% of the EU's heating energy needs by 2030 rising to $50 \%$ by 2050 and the E2District system will contribute to achieving this target. Widespread adoption of DHC systems in Europe will lead to cost savings of approx. 14 billion, the creation of an additional 220,000 jobs and further integration of RES by 2050 [5].

\section{InDeal}

InDeal Project foresees the development of an innovative platform that will automate the DHCS production planning and also will impose a fairly distribution of energy among the network's buildings by prioritizing the distribution of heating or cooling to buildings with low energy efficiency and maximum energy demand. To achieve these objectives, an advanced Decision and Support System (DSS) will monitor 24/7 the DHC system and will handle the information from the various supporting tools, such as: (i) real time energy consumption data gathering via artificial intelligent meters, (ii) forecasting and identifying the (heating and cooling) energy demand from various buildings types, depends on their energy efficiency and energy consumption, (iii) prediction of weather conditions (long-term and short-term) and iv) monitoring, control and optimal operation of the network's Thermal Energy Storage (TES). The modelling and control framework is based on a distributed, multi-layer, hierarchical architecture that is spread among three layers, the SuperStructure Model (upper layer) takes into account the physical layer description of the system and handles the strategic decisions, e.g., production planning, optimal operation of thermal storages and system level functionalities. In the medium layer we have the Slow-time scale model that models the slow dynamics of the system and coordinates the energy needs, i.e., provide the set-points to the Fasttime scale Model (lower layer) that contains the lower level controllers and the (local) process regulators (pumps, valves). The overall solution will be validated and demonstrated via two real case studies, a DH network in Vransko (Slovenia) based on biomass and a DHC network in Montpelier (France). The InDeal project solution is expected to contribute to the key objectives of the European energy research policy by promoting sustainable development, ensuring security and diversity of energy supply, improving energy efficiency and increasing industrial competitiveness and also is in alignment with the European Commission target for reduction of greenhouse gases [6].

\section{H-Disnet (H-Disnet Hybrid Thermo-Chemical Energy Networks)}

For initial stage, existing sources of residual heat (e.g., from larger heat pumps and refrigeration units) can be used for regeneration of liquid desiccants. The desiccant concentrate (a thermo-chemical fluid) then is used as an energy carrier and is transported to neighboured consumers through small scale networks. The concentrate can be used for improved performance of cooling and heating procedures. Further applications are related to industrial drying (loundry, textiles, paper, etc.). For cooling, supply air is de-humidified, lowering the total primary energy demand of AC devices. For heating, indoor room air de-humidification and reduction of pollutants (dust, VOCs) is provided in direct contact between desiccant and air, allowing for reduced fresh air ventilation and related 
reduced primary energy consumption. Demand for desiccants in the heating period is relatively low. Regeneration can be provided from fewer points, where annually heat disposal is given (industrial process heat, data centers etc.), while for cooling also hot exhaust air from AC devices can be used. Once, the technology is established, it can be extended to combined desiccant/cooling/heating networks. End user heat pumps are replaced by larger, more efficient units in the neighbourhood. Still they provide heat and cool while in summer also desiccant regeneration under use of heat pump exhaust heat is provided. In daytime, a part of rejected heat is directed to a thermal storage and can be used for desiccant regeneration during nighttime, thus allowing a $24 \mathrm{~h}$ regeneration process. For higher stability, several small scale neighbourhood networks can be connected by desiccant pipes for equalisation of differing de-humidificatiton demands especially during heating periods [7].

\section{SDHp2m (Solar District Heating: Supplying Renewable Zero-Emission Heat)}

SDHp2m is a H2020 European Project that addresses the market uptake challenges for a wider use of district heating and cooling systems (DHC) with shares of RES, especially the action focuses on the use of large-scale solar thermal plants combined with other RES in DHC systems. The project involves 15 partners from 9 different European countries and engages 9 EU regions: Styria (Austria), Thuringia (Germany), Rhone-Alpes (France), Varna (Bulgaria), Venetto and Valle d'Aosta (Italy), Västra Götaland (Sweden), Mazowsze (Poland) and Hamburg (Germany). The aims of the project are: (i) mobilize direct investments in $\mathrm{SDH}$, hence a significant market rollout, (ii) develop and implement advanced policy and (iii) support measures for SDH. Considering these goals, the project work program focuses in the selected regions by, on one hand, designing a strategy and action planning for each region based on a survey, best practices and stakeholder consultation and, in the other hand, including an implementation phase starting at an early project stage. The project also centres its work at the European level by developing an efficient dissemination of the project results, examples of best regional cases, transfer of know-how and by stimulating replication, to ensure favourable regional and European policy framework. Solar District Heating is a technology that supplies emission-free and $100 \%$ renewable energy with a cost stable for over 25 years, using a natural source, solar energy, that is unlimited and available everywhere [8].

Acknowledgments: INDIGO has received funding from European Union's Horizon 2020 research and innovation programme under grant agreement No. 696098. Flexynets has received funding from European Union's Horizon 2020 research and innovation programme under grant agreement No. 649820. E2District has received funding from European Union's Horizon 2020 research and innovation programme under grant agreement No. 696009. InDeal has received funding from European Union's Horizon 2020 research and innovation programme under grant agreement No. 696174. H-Disnet has received funding from European Union's Horizon 2020 research and innovation programme under grant agreement No. 695780. SDHp2m has received funding from European Union's Horizon 2020 research and innovation programme under grant agreement No. 691624.

Author Contributions: Andrea Costa coordinated the workshop and contributed to the INDIGO project. Susana Lopez, Tatiana Loureiro, and Francesco Passerini contributed to the INDIGO project. Dirk Pietrushka contributed to the Flexynets project. Martin Klepal contributed to the E2District project. George Halikias, Ioannis Meintanis, Serafeim Moustakidis and Nikos Karcanias contributed to the InDeal project. Martin Bucholz contributed to the H-Disnet project. Inés Arias contributed to the SDHp2m project.

Conflicts of Interest: The authors declare no conflict of interest

\section{References:}

1. European Commission. Overview of Support Activities and Projects of the European Union on Energy Efficiency and Renewable Energy in the Heating \& Cooling Sector. 2016. Available online: https://ec.europa.eu/energy/sites/ener/files/documents/overview_of_eu_support_activities_to_h-c_-_final.pdf (accessed on 3 November 2017).

2. District Cooling Open Source Library (DCOL). Available online: https://zenodo.org/record/818289\#. WgHlO7ZDlaU (accessed on 3 November 2017). 
3. INDIGO. Available online: www.indigo-project.eu (accessed on 3 November 2017).

4. FLEXYNETS. Available online: www.flexynets.eu (accessed on 3 November 2017).

5. E2District. Available online: www.e2district.eu (accessed on 3 November 2017).

6. InDeal. Available online: www.indeal-project.eu (accessed on 3 November 2017).

7. H-DisNet. Available online: www.h-disnet.eu (accessed on 3 November 2017).

8. Solar District Heating. Available online: www.solar-district-heating.eu (accessed on 3 November 2017).

(c)

(C) 2017 by the authors. Licensee MDPI, Basel, Switzerland. This article is an open access article distributed under the terms and conditions of the Creative Commons Attribution (CC BY) license (http://creativecommons.org/licenses/by/4.0/). 